\title{
Virtual reality and the new psychophysics
}

\author{
Beatrice de Gelder ${ }^{1,2}$, Jari Kätsyri' ${ }^{1}$ \& Aline W. de Borst ${ }^{2}$ \\ 1. Brain and Emotion Lab, Department of Cognitive Neuroscience, Faculty of Psychology and Neuroscience, \\ Maastricht University, Maastricht, the Netherlands. \\ 2. Department of Computer Science, University College London, London, United Kingdom.
}

\begin{abstract}
Virtual reality (VR) promises methodological rigour with the extra benefit of allowing us to study the contextdependent behaviour of individuals in their natural environment. Pan and Hamilton (2018, Br. J. Psychol.) provide a useful overview of methodological recommendations for using VR. Here, we highlight some other aspects of the use of VR. Our first argument is that VR can be useful by virtue of its differences from the normal perceptual environment. That is, by virtue of its relative non-realism and poverty of its perceptual elements, it can actually offer increased clarity with respect to the features of interest for the researcher. Our second argument is that VR exerts its measurable influence more by eliciting an acceptance of the virtual world (i.e., 'suspension of disbelief') rather than by eliciting a true belief of the realism of the VR environment. We conclude by providing a novel suggestion for combining neuroimaging methods with embodied VR that relies on the suspension of disbelief.
\end{abstract}

Using controlled but simplified stimuli has long been the method of choice in experimental psychology, as it allows for specific hypothesis testing under fully controlled laboratory conditions. However, the generalizability of findings to natural contexts could at times be called into question. Virtual reality (VR) can offer similar methodological rigour with the extra benefit of allowing us to study the context-dependent behaviour of individuals in their natural environment. Notwithstanding all the technological innovations on which it builds, one can view VR as a continuation of a long psychophysical tradition that attempts to interfere with our perception in order to clarify its underlying mechanisms. For example, lenses or prisms have been used to invert visual perception of orientation in order to study the functional properties of the visual cortex, and temporal asynchrony between audition and vision has been used to study multisensory perception. We suggest that VR is not dissimilar to such methods, except for being more radical by exchanging the normal perceptual environment with a completely virtual one.

Pan and Hamilton (2018) provide a useful overview of methodological recommendations for using VR in social interaction research and discuss a number of challenges VR is still facing. Overall, they emphasize the value of VR settings with ever greater realism and interactive responsiveness. We agree that highly realistic settings might be required for studying some research questions. However, here we want to advance two opposite arguments for using limited realism in VR, by emphasizing what VR is not, rather than what it should try to be. Our first argument is that VR can be useful by virtue of its differences from the normal perceptual environment. That is, by virtue of its relative non-realism and poverty of its perceptual elements, it can actually offer increased clarity with respect to the features of interest for the researcher. Our second argument is that VR exerts its measurable influence more by eliciting an acceptance of the virtual world (i.e., 'suspension of disbelief') rather than by eliciting a true belief of the realism of the VR environment. We conclude by providing a novel suggestion for combining neuroimaging methods with embodied VR that relies on the suspension of disbelief. 


\section{Methodological advantages of limited realism.}

Arguments in favour of adopting VR are typically based on the promises of more and more realism. VR technology will become increasingly useful for research, as it delivers more and more realistic displays. But this view assumes a simplified notion of perceptual realism of the human visual (and more generally sensory) system that is not warranted by empirical findings. The visual system is highly creative in many different ways and perception is often going beyond what is strictly physically given. A realistic representation is the result of how the visual system and higher cognitive processes work together. The perceptual system continuously fills in and constructs its reality combining what is in the mind with what the senses relay.

In a similar vein, interaction between the sensory systems and higher cognition takes place during VR experience. Capitalizing on the poverty of the VR world rather than on its richness actually offers a unique chance for investigating the design and mechanism of brain systems that underlie for example social interaction and their detailed characteristics. For example, a social agent can be designed for use in VR that is systematically different from a real life agent. Rather than striving for increased verisimilitude, researchers can create various 'impoverished' versions of real agents by systematically manipulating their attributes. The investigation of these manipulations is tantamount to developing and testing a set of hypotheses to tease apart the properties of the underlying functional design of social agency and its brain-body underpinnings.

\section{Theoretical advantages of virtual beliefs.}

The second aspect of VR overlooked in the progress towards more realism is centred on the user. The epistemological status of the VR experience has not received much attention as of yet, but it may hold a key to the usefulness of VR as a research tool, especially in studies of affect and social interaction. The most relevant aspect of VR and what makes it such a potentially useful methodology is that the user is in a state of 'suspension of disbelief', or a state where knowledge of the unreality of the VR world and belief in its experiential reality coexists. The notion of suspension of disbelief we propose here does not refer to the vividness of what is felt, or to the experience of 'presence' (Sanchez-Vives \& Slater, 2005), but it denotes the special cognitive or epistemological status of the VR experience. Although participants typically behave similarly in VR to how they would behave in a comparable situation in reality (Slater, 2009; Slater et al., 2006), they know that what happens to them in VR is not real in the same sense to that what happens outside VR is. Consequently, they do not confuse reality inside and outside VR. This situation is no different from what happens in the theatre or the movies. Readers of fiction or audiences in the theatre are willing to go along with the author in accepting the fictitious reality while letting the images and the storytelling play havoc with their emotions. The knowledge that one is in VR can be counterbalanced by the impact of the VR experience, allowing emotion systems in the brain to run free from cognitive supervision. Once the participant enters the VR, strong emotions can be triggered and spontaneous actions performed none of which necessitate a belief in the realism of the environment. This dual reality of the affective experience in VR, for example, when experiencing strong emotions while being attacked by a spider while knowing on some level that one is not in danger, ties in with a kind of dual system view on emotions in the brain which many emotion researchers now hold. This view also stresses the consciousness-independent, unintentional, and automatic nature of emotional 
processes. From an evolutionary perspective, this level of autonomic affective experience is crucial for understanding emotions, and this is one important theoretical issue that the use of VR can offer a novel view on.

Keeping at moderate levels of realism may also solve a potential challenge that the uncanny valley hypothesis (Mori, 2012) poses for virtual characters. Mori's original essay on uncanny valley implied that near-human virtual characters would inevitably elicit negative affective reactions characterized by eeriness and aversion. The bulk of empirical evidence seems to suggest that eeriness is elicited by realism inconsistency rather than human-likeness per se (for reviews, see de Borst \& de Gelder, 2015; Kätsyri, Förger, Mäkäräinen, \& Takala, 2015; MacDorman \& Chattopadhyay, 2016). This kind of realism inconsistency is not only restricted to a disparity between appearance and motion, however, but could also reflect disparities for example between static facial features (e.g. artificial eyes on a realistic face; Seyama \& Nagayama, 2007) or between face and voice realism levels (Mitchell et al., 2011). Inconsistency between appearance and behaviour might also be relevant (e.g. see 'synergy ridge' as proposed by MacDorman, Minato, Itakura, Cowley, \& Ishiguro, 2005). For example, machines that are able to feel, and humans that are not, are considered eerie at a conceptual level (Gray \& Wegner, 2012). This may mean that the 'Mons Olympus' challenge of building a fully interactive virtual human, as posed by Pan \& Hamilton (2018), would actually require a virtual character that not only acts but also looks fully human.

\section{Implementation of VR in neuroimaging experiments}

The use of VR is increasingly widespread in many domains with practical applications and has shown its strength in clinical applications (Seinfeld et al., 2018). However, we currently do not yet understand what its transformative power is based on and how it operates at the level of brain processes. As Pan \& Hamilton (2018) mention, it is not easy to combine immersive displays with neuroimaging methods. Some of the earliest studies that combined VR with neuroimaging investigated spatial cognition. Navigation through the virtual environment was usually performed with keyboard keys, or joysticks. In navigation by driving, using sets of joysticks is not that different from using the steering wheel of a car (Spiers \& Maguire, 2006), but in navigation by walking the type of movements performed during the experiment (pushing buttons) is very different from the leg movements performed during real life navigation (Burgess, Maguire, Spiers, \& O'Keefe, 2001). Using external devices, such as joysticks or button boxes, rather than using the own body to navigate through the environment may lead to a disembodied state. The lack of embodiment may impact the experience of presence in the virtual world and affect interaction and cognitive processing (Steed, Pan, Zisch, \& Steptoe, 2016). Especially in experiments on human social interaction embodiment is an important factor. Neuroimaging methods with high spatial resolution, such as functional MRI (fMRI), do not allow for much body movement, as the head needs to stay in the exact same location during measurements. Therefore, body ownership over a whole virtual body is difficult to obtain using visuoproprioception synchrony or visuo-motor synchrony as these methods require the subject to move their body. Visuo-tactile synchrony has been used to acquire ownership over another body using a video camera set-up (Guterstam, Bjornsdotter, Gentile, \& Ehrsson, 2015) and could be similarly implemented to obtain ownership over a whole virtual body. Alternatively, we 
propose that body ownership over a virtual body can also be obtained outside of the MRI scanner and can be maintained during transfer to the MRI scanner to be used as a form of priming. In a recent experiment, de Borst, Sanchez-Vives, Slater, and de Gelder (2017) showed that visuo-motor training from a first person perspective preceding $f M R I$ measurements can lead to increased plausibility, body ownership and identification with the virtual body during fMRI measurements. Participants performed visuo-motor exercises using a virtual mirror either from a first person perspective where the virtual body moved consistent with their movements, or from a third person perspective, where the virtual camera moved consistent with their movements. Afterwards, during fMRI measurements, they viewed an identical social emotional scenario. The first versus the third person perspective did not only induce differences in plausibility, body ownership and identification, but also had direct impact on brain activity during the experience of the social scenario. These results indicate that embodiment can be maintained over time and can be used, for example, to manipulate perspective during social situations.

The introduction of VR technology as part of neuroimaging experiments also creates an additional challenge - that of the analysis of data obtained in a realistic setting. If VR is indeed used to study human social interaction in an ecologically valid situation this implies that we should let go of the tradition to present the same stimulus over and over again. This, however, causes a problem for the most commonly used statistical analyses that rely on repeated stimulus presentations. Not long ago, in the field of neurocinematics, Hasson et al. (2004) suggested a new way of analysing brain data obtained during the viewing of naturalistic stimuli, such as film. Intersubject correlation is a method that measures the synchronized brain activity across subjects over time and has since been applied to social neuroscience experiments using film (Hasson et al., 2009; Hasson, Ghazanfar, Galantucci, Garrod, \& Keysers, 2012; Nummenmaa et al., 2012; Salmi et al., 2014). Similarly, this method may be applied to data collected using the VR-fMRI set-up described above, where brain responses to an identical naturalistic stimulus are measured in all subjects. Alternatively, other naturalistic techniques (e.g. Lahnakoski et al., 2012) may provide additional options in designing and analysing combined neuroimaging data and individualized VR stimuli. We may also borrow techniques from real-time fMRI, which have mainly focused on communication devices for locked-in patients (Sorger, Reithler, Dahmen, \& Goebel, 2012; Weiskopf et al., 2003), to further develop interactive VR set-ups that can be combined with neuroimaging methods (see also Tikka et al., 2012).

In conclusion, the usefulness of VR as a research tool should not be confined just to its realism. Instead, we may also benefit from its relative impoverished features to investigate brain mechanisms of sensory systems, affective processing and social interaction. Even if VR is more of an illusionary world than a perfect copy of reality, we can still make use of the effect that this illusion has on people that makes them respond very similarly to a real situation. In fact, we can make use of illusions of presence, plausibility and body ownership in VR to study the brain basis of social and affective processes. 


\section{Acknowledgements}

This work was supported by FP7/2007-2013, ERC grant agreement number 295673 to BdG and AdB, and H2020-MSCA-IF-2015 grant agreement 703493 to JK.

\section{References}

Burgess, N., Maguire, E. A., Spiers, H. J., \& O'Keefe, J. (2001). A temporoparietal and prefrontal network for retrieving the spatial context of lifelike events. Neuroimage, 14(2), 439-453. doi: 10.1006/nimg.2001.0806

de Borst, A. W., \& de Gelder, B. (2015). Is it the real deal? Perception of virtual characters versus humans: an affective cognitive neuroscience perspective. Front Psychol, 6, 576. doi: 10.3389/fpsyg.2015.00576

de Borst, A. W., Sanchez-Vives, M. V., Slater, M., \& de Gelder, B. (2017). First person experience of virtual threat enhances brain activity in the human peripersonal space network. Program No. 458.02. Neuroscience 2017. Washington, DC: Society for Neuroscience.

Gray, K., \& Wegner, D. M. (2012). Feeling robots and human zombies: mind perception and the uncanny valley. Cognition, 125(1), 125-130. doi: 10.1016/j.cognition.2012.06.007

Guterstam, A., Bjornsdotter, M., Gentile, G., \& Ehrsson, H. H. (2015). Posterior cingulate cortex integrates the senses of self-location and body ownership. Curr Biol, 25(11), 1416-1425. doi: 10.1016/j.cub.2015.03.059

Hasson, U., Avidan, G., Gelbard, H., Vallines, I., Harel, M., Minshew, N., \& Behrmann, M. (2009). Shared and idiosyncratic cortical activation patterns in autism revealed under continuous real-life viewing conditions. Autism Res, 2(4), 220-231. doi: 10.1002/aur.89

Hasson, U., Ghazanfar, A. A., Galantucci, B., Garrod, S., \& Keysers, C. (2012). Brain-to-brain coupling: a mechanism for creating and sharing a social world. Trends Cogn Sci, 16(2), 114-121. doi: 10.1016/j.tics.2011.12.007

Hasson, U., Nir, Y., Levy, I., Fuhrmann, G., \& Malach, R. (2004). Intersubject synchronization of cortical activity during natural vision. Science, 303(5664), 1634-1640. doi: 10.1126/science.1089506

Kätsyri, J., Förger, K., Mäkäräinen, M., \& Takala, T. (2015). A review of empirical evidence on different uncanny valley hypotheses: support for perceptual mismatch as one road to the valley of eeriness. Front Psychol, 6, 390. doi: 10.3389/fpsyg.2015.00390

Lahnakoski, J. M., Glerean, E., Salmi, J., Jaaskelainen, I. P., Sams, M., Hari, R., \& Nummenmaa, L. (2012). Naturalistic FMRI mapping reveals superior temporal sulcus as the hub for the distributed brain network for social perception. Front Hum Neurosci, 6, 233. doi: 10.3389/fnhum.2012.00233

MacDorman, K. F., \& Chattopadhyay, D. (2016). Reducing consistency in human realism increases the uncanny valley effect; increasing category uncertainty does not. Cognition, 146, 190-205. doi: 10.1016/j.cognition.2015.09.019

MacDorman, K. F., Minato, T., Itakura, S., Cowley, S., \& Ishiguro, H. (2005). Assessing human likeness by eye contact in an android testbed. Proc. ICCS/Cog-Sci, 1373-1378.

Mitchell, W. J., Szerszen, K. A., Sr., Lu, A. S., Schermerhorn, P. W., Scheutz, M., \& Macdorman, K. F. (2011). A mismatch in the human realism of face and voice produces an uncanny valley. Iperception, 2(1), 10-12. doi: 10.1068/i0415

Mori, M. (2012). The Uncanny Valley. leee Robotics \& Automation Magazine, 19(2), 98-100. doi: 10.1109/Mra.2012.2192811

Nummenmaa, L., Glerean, E., Viinikainen, M., Jaaskelainen, I. P., Hari, R., \& Sams, M. (2012). Emotions promote social interaction by synchronizing brain activity across individuals. Proc Natl Acad Sci U S A, 109(24), 9599-9604. doi: 10.1073/pnas.1206095109

Pan, X., \& Hamilton, A. F. C. (2018). Why and how to use virtual reality to study human social interaction: The challenges of exploring a new research landscape. Br J Psychol. doi: 10.1111/bjop.12290

Salmi, J., Glerean, E., Jaaskelainen, I. P., Lahnakoski, J. M., Kettunen, J., Lampinen, J., . . Sams, M. (2014). Posterior parietal cortex activity reflects the significance of others' actions during natural viewing. Hum Brain Mapp, 35(9), 4767-4776. doi: 10.1002/hbm.22510

Sanchez-Vives, M. V., \& Slater, M. (2005). From presence to consciousness through virtual reality. Nat Rev Neurosci, 6(4), 332-339. doi: 10.1038/nrn1651 
Seinfeld, S., Arroyo-Palacios, J., Iruretagoyena, G., Hortensius, R., Zapata, L. E., Borland, D., . . . Sanchez-Vives, M. V. (2018). Offenders become the victim in virtual reality: impact of changing perspective in domestic violence. Sci Rep, 8(1), 2692. doi: 10.1038/s41598-018-19987-7

Seyama, J., \& Nagayama, R. S. (2007). The Uncanny Valley: Effect of realism on the impression of artificial human faces. Presence-Teleoperators and Virtual Environments, 16(4), 337-351. doi: DOI 10.1162/pres.16.4.337

Slater, M. (2009). Place illusion and plausibility can lead to realistic behaviour in immersive virtual environments. Philosophical Transactions of the Royal Society B-Biological Sciences, 364(1535), 3549-3557. doi: 10.1098/rstb.2009.0138

Slater, M., Antley, A., Davison, A., Swapp, D., Guger, C., Barker, C., . . Sanchez-Vives, M. V. (2006). A Virtual Reprise of the Stanley Milgram Obedience Experiments. Plos One, 1(1). doi: ARTN e39 10.1371/journal.pone.0000039

Sorger, B., Reithler, J., Dahmen, B., \& Goebel, R. (2012). A Real-Time fMRI-Based Spelling Device Immediately Enabling Robust Motor-Independent Communication. Current Biology, 22(14), 13331338. doi: 10.1016/j.cub.2012.05.022

Spiers, H. J., \& Maguire, E. A. (2006). Thoughts, behaviour, and brain dynamics during navigation in the real world. Neuroimage, 31(4), 1826-1840. doi: 10.1016/j.neuroimage.2006.01.037

Steed, A., Pan, Y., Zisch, F., \& Steptoe, W. (2016). The Impact of a Self-Avatar on Cognitive Load in Immersive Virtual Reality. 2016 leee Virtual Reality Conference (Vr), 67-76.

Tikka, P., Valjamae, A., de Borst, A. W., Pugliese, R., Ravaja, N., Kaipainen, M., \& Takala, T. (2012). Enactive cinema paves way for understanding complex real-time social interaction in neuroimaging experiments. Front Hum Neurosci, 6. doi: Artn 298 10.3389/Fnhum.2012.00298

Weiskopf, N., Veit, R., Erb, M., Mathiak, K., Grodd, W., Goebel, R., \& Birbaumer, N. (2003). Physiological self-regulation of regional brain activity using real-time functional magnetic resonance imaging (fMRI): methodology and exemplary data. Neuroimage, 19(3), 577-586. doi: 10.1016/S10538119(03)00145-9 